INVESTIGACIÓN/RESEARCH

Recibido: 20/05/2014---Aceptado: 03/06/2014---Publicado: 15/06/2014

Claudia López Frías1: Universidad Complutense de Madrid (España)

Claudia.lopez8@hotmail.com

\title{
LA INTERRELACIÓN ENTRE LAS REDES SOCIALES ESPECÍFICAS DE LA COMUNICACIÓN CIENTÍFICA Y LAS REDES SOCIALES DE USO GENERAL.
}

\section{RESUMEN}

Actualmente estamos inmersos en una era de cambios, donde las tecnologías de la Información y la Comunicación están renovando todos los ámbitos de nuestra vida. En el ámbito científico el cambio íntimamente relacionado con las redes sociales, tanto las de uso general como las redes sociales específicas de la ciencia. Si en un principio se idearon como una moda adolescente actualmente se está convirtiendo en una herramienta que empieza a revolucionar la manera en la que se divulgan los resultados científicos. Uno de los objetivos más primordiales de las investigaciones es llegar hasta un gran número de ciudadanos debemos para ello interrelacionar más las redes sociales específicas de comunicaciones científicas con las redes sociales de uso general.

\section{PALABRAS CLAVE}

Redes Sociales - Investigación - Comunicaciones - Nuevas Tecnologías Colaboración Científica.

1 Claudia López Frías: doctoranda en Comunicación Audiovisual y Publicidad en la Universidad Complutense de Madrid. Realizadora y cámara en Canal Senado Televisión. Sus principales líneas de investigación son el cine y su evolución. 
Redes Sociales - Investigación - Comunicaciones - Nuevas Tecnologías -

Colaboración Científica

\title{
THE INTER-RELATION BETWEEN SPECIFIC SOCIAL NETWORKS FOR SCIENTIFIC COMMUNICATION AND GENERAL USE SOCIAL NETWORKS.
}

\begin{abstract}
Currently we are immersed in an era of changes, where the technologies of information and communication are changing all areas of our lives. In the field of science the change is closely linked with social networks, both of general use as well as the specific social networks of the field. If at the beginning they were designed as a teenage trend, now they are becoming a tool that is revolutionizing the way in which scientific results are made public. One of the basic goals of the investigations is to reach a great number of citizens, for this we must interrelate the more science-specific social networks with the general use ones.
\end{abstract}

\section{KEYKORDS}

Social Network - Investigation - Communications - New Technologies - Scientific Collaboration.

\section{INTRODUCCIÓN}

Recientemente estamos viviendo una era de cambios en el que el sector que no se renueva pasa a un segundo plano, y puede perder el espacio, profesional o personal, que estaba destinado a él. Es por ello imprescindible cambiar nuestras rutinas y adaptarnos a las novedades que han traído consigo las nuevas tecnologías de la información y la comunicación. Lo mismo ocurre en el sector científico, en los dispositivos a los que los científicos acuden para mostrar los resultados de sus investigaciones podemos encontrar que cada vez son más en formato electrónico. Siempre han estado en manos de grupos de personas con intereses comunes, que trabajaban en forma de red dentro de un mismo ámbito, colaborando entre ellos y favoreciendo al grupo mediante citas y demás. La herramienta para divulgar sus resultados eran tanto las revistas como el papel, ambas, naturalmente, siguen teniendo un papel fundamental hoy en día, pero los beneficios de la utilización de Internet para la divulgación de estos resultados no se han hecho esperar.

El primero de estos beneficios es la disolución de las fronteras espaciales. Cualquier persona desde cualquier sitio le basta tener un ordenador con acceso a internet para poder acudir a cualquier investigación o publicar la suya. Por otro lado, también se 
puede apreciar una disolución de las fronteras institucionales, ya que no es necesario estar vinculado a un colectivo para poder divulgar tu estudio. La suma de estos dos beneficios ha traído como consecuencia el tercer beneficio, se trata del incremento de investigaciones que son colaboraciones de científicos de distintas latitudes y que perteneces a instituciones diferentes. Esto es algo tremendamente beneficioso para la ciencia, ya que este tipo de colaboraciones suele tener como resultado estudios brillantes.

Naturalmente todo esto trae como consecuencia uno de los mayores beneficios, se trata, sin duda, de un incremento en la difusión y divulgación de los conocimientos. Sin embargo aún es necesario dar un paso más y estimular a los jóvenes a interesarse por la divulgación científica así como involucrar más a los ciudadanos en la investigación. Para ello debemos sacar el máximo provecho a las nuevas tecnologías teniendo en cuenta que la utilización de las redes sociales puede ser la herramienta perfecta tanto para divulgar el conocimiento como para encontrar oportunidades.

Es fundamental tener una imagen correcta en las redes sociales, por supuesto tener un perfil, ya que actualmente, quien no está en las redes sociales no existe. Lo que en un principio puede parecernos una moda adolescente es en realidad una herramienta que está en pleno cambio para revolucionar el mundo de la divulgación científica. Por un lado las propias redes sociales específicas de investigación como Reserach Gate, BiomedExpert o Reisearch son la herramienta perfecta para agilizar la comunicación científica, facilitando el trabajo a los investigadores y con una serie increíble de ventajas como puede ser la estimulación de la creatividad en grupo o el acceso intersectorial. Las redes sociales generales, como Facebook por ejemplo, son eficaces reuniendo gente de distintos puntos y poniéndolas en contacto.

Pero... ¿dónde empieza exactamente y dónde termina una red social? El mundo en el que nos movemos son redes sociales, el gobierno, las instituciones e incluso nosotros mismos somos redes sociales que interactuamos y nos asociamos con otras redes para hacer crecer la nuestra. En este trabajo por redes sociales nos referiremos a la estructura por la cual una persona o grupo de personas están interrelacionadas mediante diferentes tipos de relaciones, es decir, estructuras de intercambio de componente social online. El uso que hacemos de estas redes sociales es cada vez de mayor transcendencia, y en los países desarrollados no se concibe una vida sin estar presente en alguna de estas redes e ir plasmando tu cotidianeidad en ellas. El uso de estas redes ha sobrepasado cualquier otra actividad online. No hay que menospreciar el poder que puedan tener estas redes sociales generales ya que han demostrado en varias ocasiones ser la herramienta más efectiva para la organización ciudadana, tal es el ejemplo de la "Acampada Sol" que comenzó como una manifestación el 15 de mayo de 2012, cuya convocatoria fue multitudinaria y se hizo a través de las redes sociales, pero en la que posteriormente, también debido a las redes sociales, cientos de personas, de distintas latitudes se reunieron durante varias semanas en la conocida plaza céntrica de Madrid para pedir un cambio político. Las redes sociales están cada vez más presentes en todos los sectores, desde una tienda hasta una biblioteca, ya que con su utilización pretenden conectar con un mayor público, 
conocer sus gustos, atraer a la gente joven y promocionar sus actividades. Es por ello primordial para el investigador tener un lugar digital donde pueda mostrar su trabajo socialmente, como podría ser una red social científica, pero también hacerlo llegar al mayor número posible de personas, como pudiera ser una red social general. El profesional debe tener un conocimiento actualizado de las redes de intercambio social con el fin de lograr una buena distribución del conocimiento y relacionarse con otros autores o recuperar relaciones de trabajo ya existentes.

\section{OBJETIVOS}

El objetivo de este estudio ha sido analizar la relación que existe entre las redes sociales específicas de la ciencia y las redes sociales de uso general así como exponen las comunicaciones científicas en ambas tecnologías. Comprobando como el publicitarse en las redes sociales de uso general puede traer un beneficio tanto a la red social científica que puede plasmarse en número mayor de investigadores que se unen en su red, pero también para el investigador, que puede divulgar su trabajo entre un mayor número de personas.

También hemos pretendido analizar la relación que los investigadores tienen con la red social científica analizando si esa relación se fortalece a través de las redes sociales de uso general.

\section{METODOLOGÍA}

La metodología utilizada consta de dos partes muy concretas. Por un lado haremos un análisis teórico de la actualidad de la cuestión para posteriormente centrarnos en un estudio empírico cualitativo donde trataremos la relación entre las redes sociales científicas y las redes sociales de uso general.

Utilizamos para la realización del trabajo la base de datos Reserach Gate, Nature Network y Mendely para comprobar cuáles de esas revistas tienen perfil en las principales redes sociales, entre las que hemos considerado Facebook, Twitter y Linkedin por ser abiertas y abarcar a un enorme número de personas.

Además veremos cómo se publican determinados artículos en las diferentes redes, y ver qué utilidad dan las redes sociales científicas a las redes de uso general, de qué manejan las utilizan. 


\section{RESULTADOS}

\section{Redes Sociales específicas de la Comunicación en la Ciencia}

El investigador tiene a su alcance una serie de herramientas que pueden serle útiles y facilitarle su trabajo. Nos referimos a las tecnologías 2.0 que permiten al investigador exponer los resultados de sus estudios de una manera abierta, y además facilita el contacto que cualquier otra persona quiera tener con él. Estas redes le dan al investigador un "algo más" que no pueden darle las redes sociales de uso general. Ese algo más es definido por Cano et. Al. Cómo: "comunidades científicas que emplean tecnologías participativas para el intercambio de información." (Cano et.al. 2010) Es decir, el uso de estas redes puede conllevar para el investigador un salto cualitativo en su investigación, al estar enriquecido con aportaciones de otros investigadores.

Además de los beneficios de los que ya hemos hablado para el investigador de este tipo de redes sociales debemos mencionar uno último: las subvenciones a proyectos de investigación encuentran en este tipo de redes una gran aliada.

Debemos mencionar que no todo son ventajas en lo referente a la Web 2.0. Ya que los debates que pudieran darse entre investigadores, aunque puedan ser muy beneficiosos para la investigación en curso suelen quedar registrados, y se hacen públicos de una manera relativamente sencilla, con lo que ese proyecto sale a la luz sin un registro de propiedad, lo cual puede causar el recelo de determinados investigadores que no les guste compartir de esa manera su trabajo. Aunque por otro lado es una consecuencia inevitable de la retroalimentación, ya que al compartir tu trabajo con otras personas para que este crezca con otros puntos de vista quedas expuesto a cambio de esa cooperación.

Una de esas redes es Research Gate Scientific Network (http://www.researchgate.net/). Fundada en mayo del 2008 por los doctores Ijad Madisch, Sören Hofmayer, Horst Fickenscher. Ahora conserva multitud de inversores, entre los que se encuentra Bill Gates. En la actualidad cuenta con más de cuatro millones de miembros que, mediante un registro gratuito, pueden compartir su trabajo así como acceder al trabajo de otros científicos, contando con una base de datos de más de 35 millones de registros. La manera en la que surgió ReSearchGate es toda una declaración de intenciones sobre sus características. "I had a research problem I couldn't solve on my own, so I started looking for other scientists who could help me out," he said. "I discovered that it's impossible using the current structure of the internet to find these people easily. "I realized science was broken," he added." (Madish 2013)

Contactar y colaborar con otros investigadores, ya sean de tu campo de estudio o de diferentes registros, y una herramienta muy útil dentro de esta página es la posibilidad de ver las estadísticas de citaciones que tiene tu trabajo, así como las visitas del mismo. Además cuenta con una bolsa de trabajo relacionada con el campo de la investigación. Por otro lado, puedes personalizar tu perfil y unirte o crear grupos de discusión respecto a un tema concreto. 
Nature Network (http:/ / network.nature.com/). Fundada en el 2007 por la editorial Nature Publishg Group actualmente cuenta con unos 25000 miembros que una vez registrados pueden comentar en foros o personalizar su perfil indicando los temas de investigación que les interesa o los proyectos que estén llevando a cabo. Cuenta además con una serie de grupos de investigación siendo el más popular Brain Physiology, Cognition and Consciousness que cuenta con 606 miembros 156 temas a debate y más de 3500 comentarios al respecto.

Mendeley (http://www.mendeley.com) Bajo el eslogan It's time to change the way we do research encontramos una red social y un gestor de referencias con la que puedes colaborar en línea con otros investigadores, encontrar otros documentos sobre el tema en el que se esté investigando en una enorme base de datos organizada por materias o por las publicaciones más buscadas, generar bibliografías automáticamente, etc., Es de descarga gratuita y permite además subir videos y otro tipo de documentos.

Fue fundada en 2007, en Londres aunque no fue publicado hasta el 2008, por Victor Henning, Paul Foeckler and Jan Reichelt y actualmente cuenta con multitud de inversores, entre los que encontramos el ejecutivo de Last Fm, los ingenieros que fundaron Skype, o académicos de Cambridge.

Cuenta además con una novedosa aplicación para iPhone con la que leer periódicos, e incluso citarlos al mismo tiempo.

Redes sociales de uso General

Como hemos visto, las redes sociales son una realidad en nuestra vida, tanto como para individuos como para organizaciones. Las posibilidades que ofrecen están cada vez más destinadas a una confluencia de servicios y aplicaciones en variedad de dispositivos. Las redes sociales son definidas por Hernández como "asociaciones de personas ligadas por motivos heterogéneos y que conforman una estructura compuesta por nodos unidos entre ellos por más de un tipo de relación" (Hernández, 2008: 30). En este sentido hay varios motivos por los que varias personas puedan estar ligadas en esta red, por ello es más factible llegar a un mayor número de personas que únicamente con una red social científica, con la que las personas están ligadas por intereses científicos. De las tres redes sociales que hablaremos a continuación todas tienen aplicaciones para poder verlas desde cualquier dispositivo móvil.

Facebook. (www.facebook.com), esta red social es tan conocida que incluso hay una película que narra sus orígenes modestos en la universidad de Harvard: La red Social (The social Network, David Ficher 2010). Actualmente para poder acceder sólo es necesario tener una cuenta de correo electrónico ya que su acceso es gratuito y completamente libre. Es una red que ha recibido bastante atención ya que terceras personas pueden crear aplicaciones y páginas para expandir su negocio. 
Twitter (www.twitter.com), es una red social basada en el microbblogin, es decir mandar mensajes cortos (140 caracteres como máximo) llamados Tweets y que aparecen en la página del usuario, y de todos aquellos que estén "suscritos" (sean seguidores) del usuario. Al mismo tiempo, el usuario puede suscribirse (hacerse seguidor) de otras personas. Debido a la limitación de caracteres se han creado toda una serie de aplicaciones que acortan las URLs, con el fin de poder compartir una página web y que aun te queden caracteres para comentarla. La que usa el propio Twitter es Bit.ly. Además hay una serie de programadores de tweets, con los que indicando el tweet y la hora y el día que quieres que se publiquen la aplicación lo llevará a cabo. Esto es extremadamente útil para empresas que utilizan la red social para publicitar sus eventos.

La última que mencionaremos será Linkedin (www.linkedin.com), una red social orientada al mundo de los negocios. Fundada en diciembre del 2002 y compuesta por millones de usuarios y millones de empresas, esta red social ayuda a los profesionales a ponerse en contacto entre ellos y generar negocios.

Analizando las relaciones entre las redes sociales científicas con las redes sociales de uso general nos damos cuentan que son más colaborativas de lo que en un principio sospechábamos. Y la participación en estas redes es cada vez mayor de hecho: "En los últimos cinco años ResearchGate, Mendeley y Academia.edu han generado individualmente una base de usuarios de casi dos millones de personas" (Mallaparti 2012)

Al iniciar sesión en Research Gate, lo podemos hacer introduciendo todos nuestros datos o simplemente iniciando sesión mediante nuestra cuenta de Facebook. De esta última manera será mucho más rápido y nos ahorraremos volver a dar nuestros datos personales.

Además Reseach Gate cuenta con página en Facebook, (facebook.com/ResearchGate) en la que tiene más de 17660 miembros con una participación bastante activa en la se publican artículos exitosos, así como noticias curiosas para la ciencia.

Con esta página se resuelven dudas sobre Researchgate.net y se le da publicidad, de manera que intenta llegar a un mayor número de personas. Así mismo se publicita su blog (https://news.researchgate.net/), de reciente creación que tiene una sección de Preguntas Frecuentes y donde se comparten todas las novedades y los cambios de la red. 


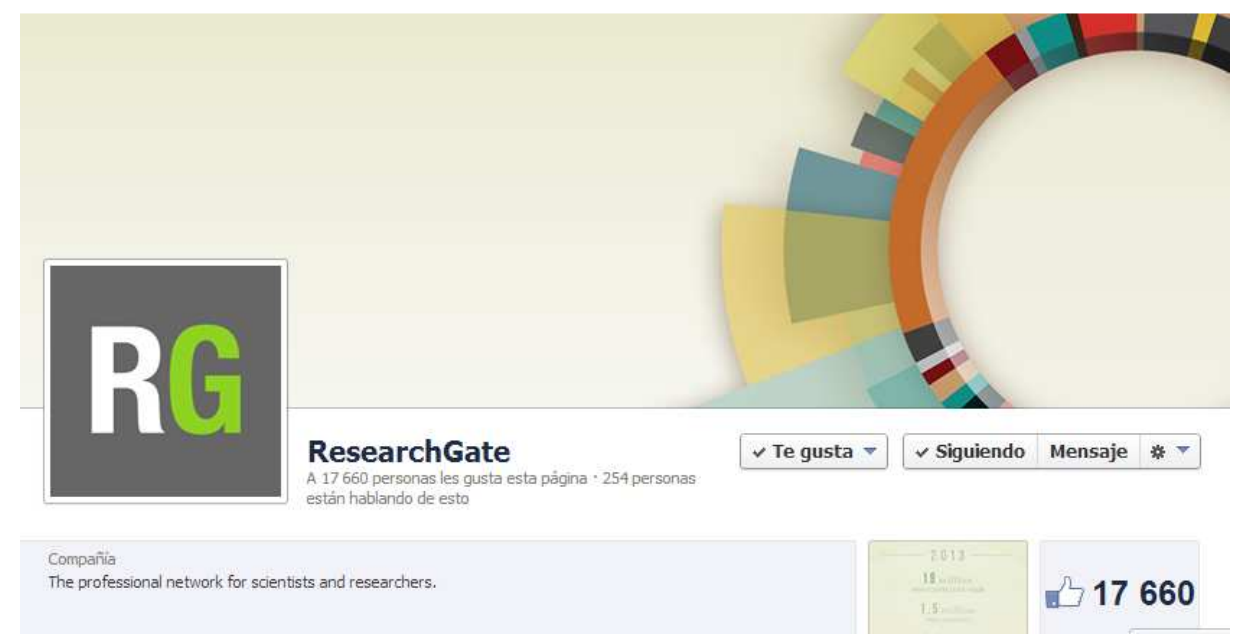

Foto 1: Portada de Facebook de Research Gate

Fuente: facebook.com/ResearchGate

En Linkedin RearchGate también tiene un perfil con 1456 seguidores. En este perfil se especifican los detalles para acceder a las demás redes sociales así como una breve descripción de en qué consiste la red social y como fue creada. Además 86 personas dentro de Linkedin han indicado que trabajan para la RearchGate o que han publicado artículos en ella, lo cual contando con que tiene una plantilla de entre 51 y 200 empleados es un número bastante significativo.

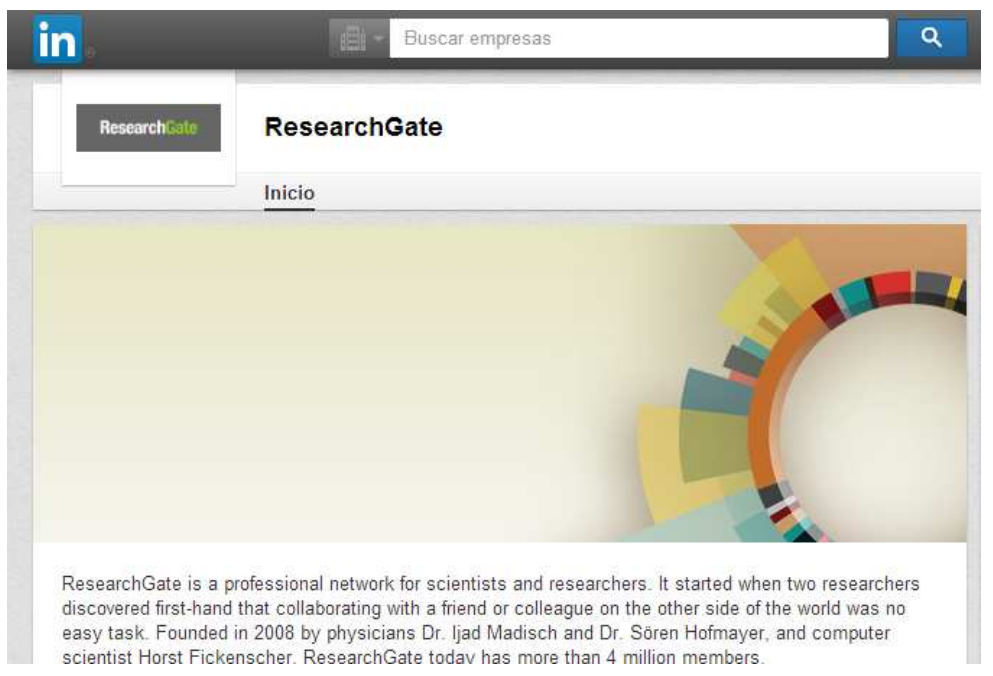

Foto 2: Portada de Linkedin de ResearchGate

Fuente: Linkedin.com/RearchGate 
Por último hemos analizado el perfil de twitter de RearchGate, (twitter.com/ResearchGate) que contiene dos cuentas, por un lado con el nombre de @ResearchGate y por otro @RSGSupport.

@ResearchGate cuenta con 10465 seguidores, y el sigue a 604. Con un total de 3403 tweets publicados en los que plantea debates, anima a unirse en las discusiones de la web e informa de noticias científicas. Por otro lado, su cuenta @RSGSupport cuenta con muchos menos seguidores (105), personas a las que sigue(21) y tweets publicados (155), pero se trata de una cuenta exclusivamente para dar apoyo e intentar solucionar los problemas que se puedan tener con la red social.

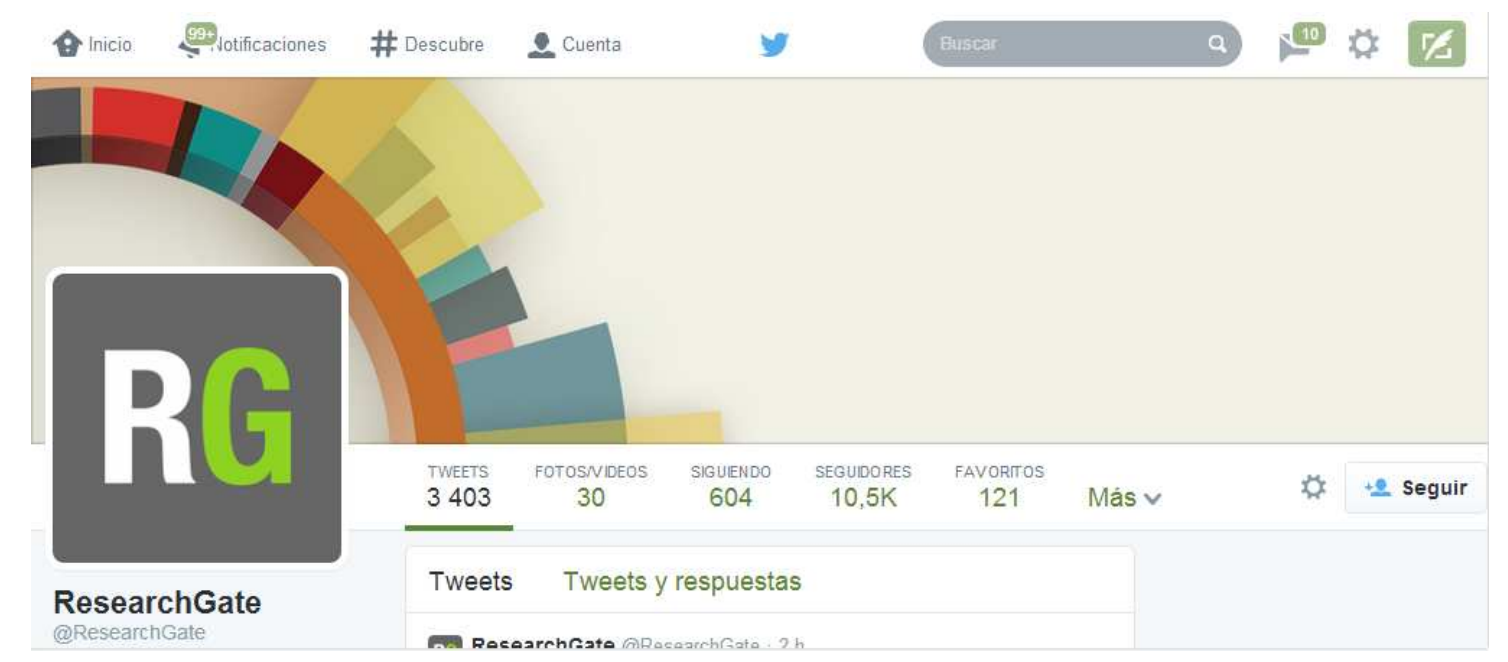

Foto 3: Portada de Twitter de ResearchGate

\section{Fuente: twitter.com/ResearchGate}

NatureNetwork no cuenta con perfiles activos en ninguna de las redes sociales analizadas, cuenta sin embargo con un blog http://blogs.nature.com/ en el que cuenta con más de veinte categorías, que es bastante activo y donde redacta asuntos que interesan a los colaboradores o dan respuesta a las peticiones de algunos investigadores.

Uno de los grupos dentro de Nature Network: Nature Network Toronto, un foro para los ciudadanos de Toronto, donde pueden discutir sobre distintos temas, $\mathrm{o}$ buscar trabajo, tienen un grupo de twitter gestionado por uno de los moderadores: Jeff Sharom. 
La última red social científica analizada, Mendeley, cuenta con perfil en todas las redes sociales analizadas. En Facebook el perfil de la red social cuenta con 20588 miembros. En su página de portada adelanta el sentido de la página "Mendeley is a free reference manager and academic social network that can help you organize your research, collaborate with others online, and discover the latest research." Comprobamos como es bastante active en Facebook donde publica por un lado las mismas cosas que en las otras redes sociales: videos sobre Mendeley, noticias sobre ciencias o las actualizaciones de su blog (blog.mendeley.com), pero también publica las experiencias de las personas que han utilizado Mendeley, que indican lo que esta red les ha ayudado en sus investigaciones.

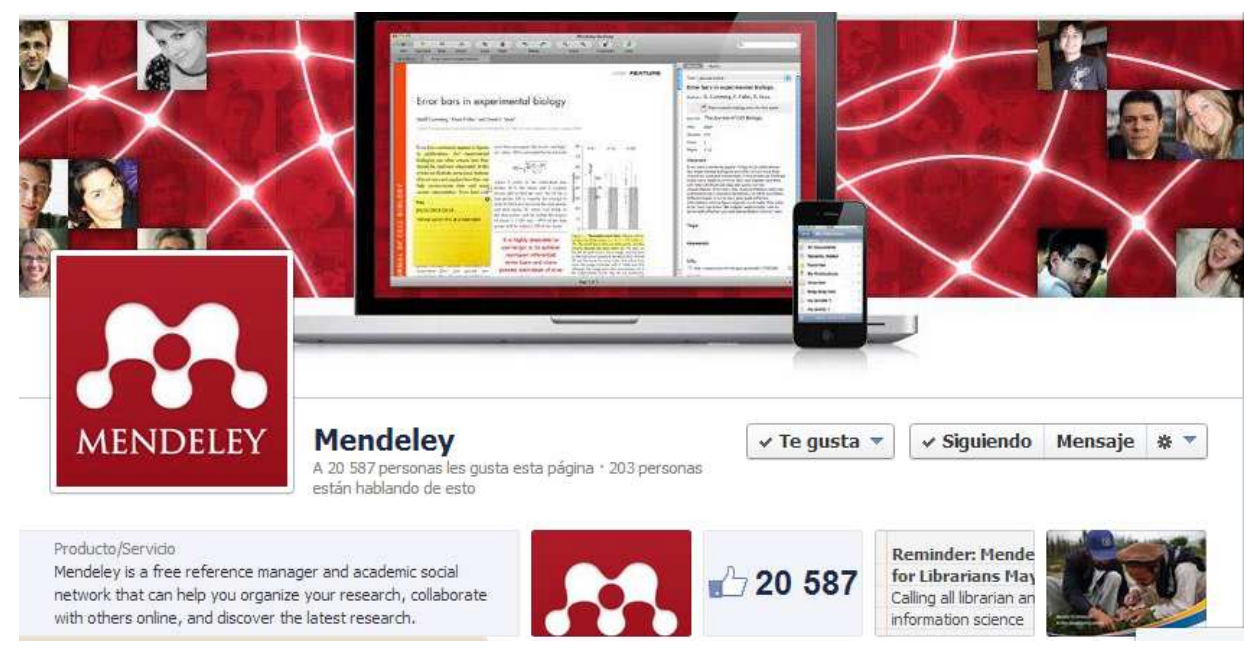

Foto 4: Portada de Facebook de Mendeley

Fuente: Facebook.com/Mendeley

En Linkedin el perfil de Mendeley, cuenta con 4399 miembros, de entre ellos 94 personas afirman estar empleados para Mendeley. En su perfil publican lo mismo que ya han publicado en Facebook o twitter, pero con la limitación de no añadir las experiencias de las personas que lo utilizan. Sin embargo puede conectarse con las personas que trabajan para la red social o entrar en contacto con aquellos que indiquen que tienen artículos publicados para Mendeley. 


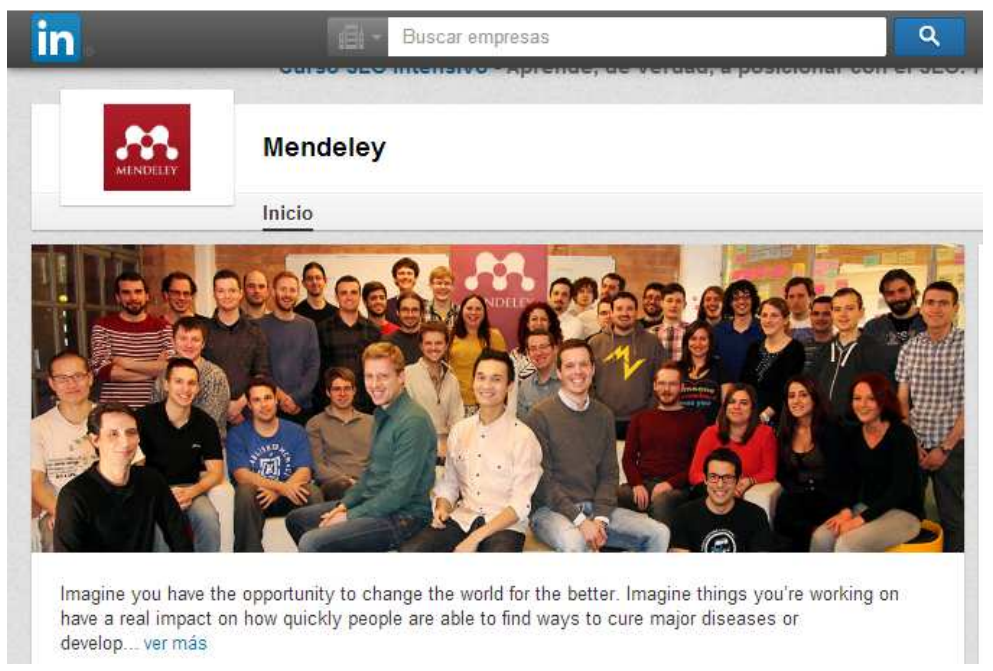

Foto 5: Portada de Linkedin de Mendeley

Fuente: Linkedin.com/Mendeley

Medeley es más activo en twitter que en cualquier otra red social, además de donde más cuenta con un "feedback" de los usuarios. Cuenta con varios perfiles:

@Mendelysupport, el primero que encontramos es un perfil creado para ayudar y dar apoyo a los investigadores que utilicen Mendely. Tiene 4812 seguidores, mientras que sólo sigue a tres personas y tiene en total 2527 tweets publicados. En este perfil responde a todos los científicos que le preguntan sus dudas y trata de resolver los problemas de estos con la página.

También encontramos los perfiles de los fundadores de Mendeley: @Mendely_com (Victor@Mendeley) que tiene 7650 seguidores, frente a los 567 que sigue y @cpf118 (Paul@Mendeley) que tiene 672 seguidores y sigue a 43, siendo mucho menos activo en la red.

@MendelyTips es el perfil oficial de Mendeley, tiene 3880 seguidores y sigue a 886, con 369 tweets. Con este perfil se intenta sacar el máximo partido a la red social, en ella da trucos para que los usuarios encuentren instrucciones precisas para algunas aplicaciones.

Otra de las cuentas es @Mendeleypoems que tiene veinte seguidores, sigue a dos personas y tiene diecinueve tweets en total. Esta página fue creada para un concurso, en la que los seguidores mostraban su amor por Mendeley con un haiku, de manera que el que más retweets tuviese ganaría un año de cuenta Premium en Mendeley durante un año. El concurso terminó en el 2011 y desde marzo de ese año no se publica nada. 
Redes Sociales - Investigación - Comunicaciones - Nuevas Tecnologías Colaboración Científica

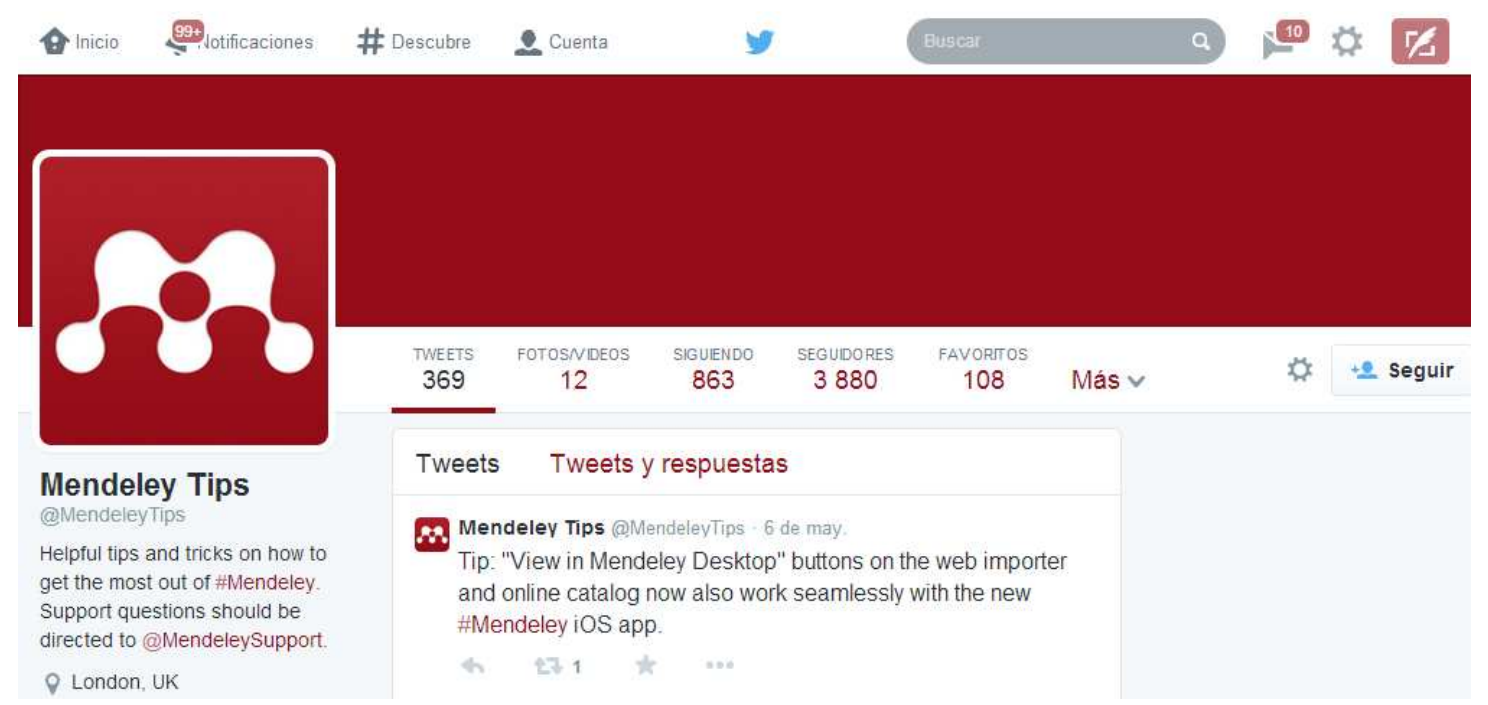

Foto 6: Portada de twitter de Mendeley

Fuente: Twitter.com/Mendeley

\section{DISCUSIÓN}

¿Cómo colaboran los científicos en un mundo globalizado? ¿Ha sacado el sector científico el máximo partido a las nuevas tecnologías? Son algunas de las preguntas que nos realizamos a la hora de comenzar este estudio.

Una vez finalizada la investigación hemos sacado algunos aspectos que consideramos claves a la hora de potenciar la investigación científica y acercarla al ciudadano.

Si bien es cierto que las redes sociales tienen un uso generalizado y masivo entre la mayoría de la población pocas veces se saca un aprovechamiento didáctico a estas redes, y su explotación formativa está aún por descubrir. Las redes sociales científicas, en cambio fomentan el aprendizaje y son auténticas redes de conocimiento cuyo uso tiene la finalidad educativa y profesional. Con la fusión de las dos redes se lograría un avance tanto a nivel cualitativo como cuantitativo para la ciencia, que pasaría de ser un tema tratado en revistas y libros especializados, como era hasta la aparición de estas redes sociales, a un tema de interés general para el ciudadano que usaría su aplicación habitual para plasmar su punto de vista sobre determinados temas o estar informado de los últimos proyectos. Así mimo su utilización en el ámbito universitario sería también tremendamente rentable para la educación ya que se lograría una comunicación más fluida entre alumnos y docentes y se llegaría a una colaboración más completa para por ejemplo, proyectos grupales. 
Este es un aspecto que nadie pasa por alto, y que ha sido muchas veces estudiado: $L a$ construcción de grupos, la conexión inmediata o el sistema descentralizado que mantienen las redes sociales han facilitado la creación natural de una inteligencia colectiva [...], un aprendizaje continúo fruto de la colaboración y la cooperación. Sistemas que son independientes, personalizados y, a la vez, tremendamente diversos. (Ortega 2008:19)

Si bien es cierto que normalmente el uso de redes sociales generales suele hacerse como un mero entretenimiento, por ello el uso de la ciencia y de los métodos de aprendizaje en estas redes debe de ser divertido y de fácil comprensión. Ese es el camino que algunas redes como Mendeley o Research Gate está haciendo de estas redes exponiendo proyectos científicos de manera amena y junto con concursos y consejos de apoyo. Otro aspecto a tener en cuenta es el componente emocional respecto a la relación entre las instituciones como Research Gate en su página de red social como Facebook o twitter y sus seguidores ya que permite establecer unos diálogos que fortalecen la relación. En este sentido es fantástica la cuenta de twitter ReseachGate Support que trata de resolver todas las dificultades y dudas que pudieran tener con la página, además de tolerar las quejas de los usuarios más disconformes. Al igual que la página de twitter Medeleypoems donde los usuarios escriben poemas que traten sobre Mendeley para ganar una cuenta Premium. De esta manera las redes sociales se hacen más cercanas a los ciudadanos que las ven más accesibles.

Por todo ello la presencia de las redes sociales científicas en las redes sociales de uso general no debería minusvalorarse, sino al contrario, considerarlo como una herramienta perfecta para la difusión masiva de determinadas investigaciones. La manera perfecta en que estas redes llegan a un mayor número de personas pareciendo más accesibles y cercanas a los ciudadanos y dándose a conocer entre un mayor número de investigadores.

\section{REFERENCIAS}

Bibliografía

Hernández Requena, S. (2008). El modelo constructivista con las nuevas tecnologías: aplicado en el proceso de aprendizaje. RUSC. Revista de Universidad y Sociedad del Conocimiento. Vol. 5, n. ${ }^{\circ}$ 2, págs. 26-35

Ortega, Gacitúa, J. C. (2008). Espacios interactivos de comunicación y aprendizaje. La construcción de identidades. RUSC. Revista de Universidad y Sociedad del Conocimiento. Vol. 5, n. ${ }^{\circ}$ 2, págs. 17-25.

\section{Referencias electrónicas}

Maestro Cano JA, Ribes Llopes I, Merlo Vega JA, Ferreras Fernández T, Gallo León JP, Angosto Castro A. (7 y 8 de octubre de 2010) Ciencia 2.0: aplicación de la Web social a 
Redes Sociales - Investigación - Comunicaciones - Nuevas Tecnologías Colaboración Científica

la investigación. X Workshop Rebiun sobre proyectos digitales: diez años de proyectos digitales: cambian las bibliotecas, cambian los profesionales. Disponible en: http://dspace.upv.es/manakin/handle/10251/8653

Consultado el 20 de abril del 2014

ORSINI, Lauren (04 de junio de 2013) Bill Gates Backs "Open Science" Social Network ResearchGate In Push For Nobel Prize. Disponible en: http:/ / readwrite.com/2013/06/04/bill-gates$\underline{\text { researchgate\#awesm }=\sim \text { oEkoBGdqgXsbx4 }}$

Consultado el 8 de mayo del 2014

MALLAPARTI Smriti (07 de noviembre 2012) Partido en el ciberespacio: La ciencia nutre a las redes. SciDevNet. Disponible en http://www.scidev.net/america$\underline{\text { latina/tic/especial/partido-en-el-ciberespacio-la-ciencia-nutre-a-las-redes.html }}$

Consultado el 24 de marzo del 2014 